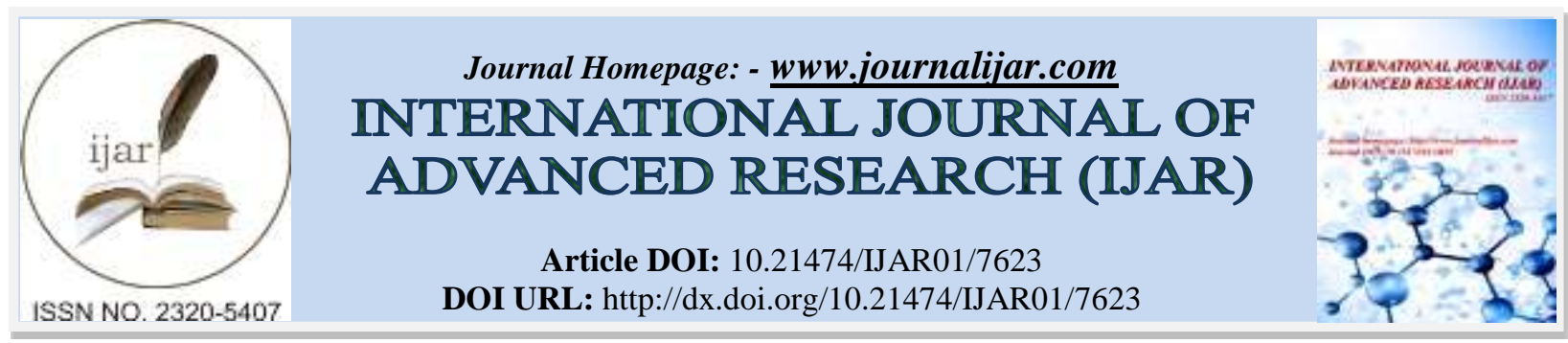

RESEARCH ARTICLE

\title{
APPLICATIONS OF GENERALIZED INVERSES BOTH IN HOMOGENEOUS AND NON- HOMOGENEOUS SYSTEM OF LINEAR EQUATIONS.
}

\begin{abstract}
Mohammad Maruf Ahmed.
Assistant Professor, Department of Mathematics and Natural Sciences, BRAC University, 66 Mohakhali Dhaka 1212, Bangladesh.
\end{abstract}

\section{Manuscript Info}

Manuscript History

Received: 23 June 2018

Final Accepted: 25 July 2018

Published: August 2018

Keywords:-

consistent, hermitian, row-echelon,

homogeneous.

\section{Abstract}

This paper deals with applications of generalized inverses (g-inverses) both in homogeneous and non-homogeneous system of linear equations. General as well as particular solutions have been derived associated with g-inverses. An example is shown which gives the general solutions of a consistent system of linear equation through the help of g-inverse.

Copy Right, IJAR, 2018,. All rights reserved.

\section{Introduction:-}

When the ordinary inverse of a square matrix cannot be used for the solution of the linear equations, we can use the g-inverse and M-P g-inverse of the singular or rectangular matrices for the solutions of the systems of linear equations.

Theorem 1. A necessary and sufficient condition for the equation $A^{+} B^{+}=C$ to have a solution is $A^{+}(A C B) B^{+}=C$ in which case the general solution is

$$
X=Y-A A^{+} Y B^{+} B+A C B \text {, where } Y \text { is arbitrary. }
$$

Proof. $C=A^{+} X B^{+}=A^{+} A A^{+} X B^{+} B B^{+}=A^{+} A C B B^{+}$

Since $A^{+} X B^{+}=C$.

Conversely, if (1) holds, then $X=A C B$ is a particular solution of $A^{+} X B^{+}=C$

Now any expression of the form $X=Y-A A^{+} Y B^{+} B$ satisfies $A^{+} X B^{+}=0$ beacause

$$
\begin{aligned}
A^{+}\left[Y-A A^{+} Y B^{+} B\right] B^{+} & =A^{+} Y B^{+}-A^{+} A A^{+} Y B^{+} B B^{+} \\
& =A^{+} Y B^{+}-A^{+} Y B^{+} \\
& =0 .
\end{aligned}
$$

The general solution of a non-homogeneous equation $A^{+} X B^{+}=C$ is equal to the general solution of $A^{+} Y B^{+}=0+$ a particular solution of $A^{+} X B^{+}=C$. Hence the general solution of $A^{+} X B^{+}=C$ is given by 
$X=Y-A A^{+} Y B^{+} B+A C B$ where $Y$ is arbitrary.

Theorem 2. If $P=\left[\begin{array}{l}A \\ B\end{array}\right]$ and $P^{-1}=[X Y]$ then $A X=I$ and $B^{+} X=0$ have $a$ unique common solution given by $X=\left(I-B B^{+}\right)\left(A-A B B^{+}\right)^{+}$.

Proof.

$$
\begin{gathered}
A X=A\left(I-B B^{+}\right)\left(A-A B B^{+}\right)^{+} \\
=\left(A-A B B^{+}\right)\left(A-A B B^{+}\right)^{+} \\
=I \\
B^{+} X=B^{+}\left(I-B B^{+}\right)\left(A-A B B^{+}\right)^{+} \\
=\left(B^{+}-B^{+} B B^{+}\right)\left(A-A B B^{+}\right)^{+} \\
=\left(B^{+}-B^{+}\right)\left(A-A B B^{+}\right)^{+} \\
=0 .
\end{gathered}
$$

Theorem 3. If $Q=[C D]$ and $Q^{-1}=\left[\begin{array}{l}Z \\ T\end{array}\right]$ then $Z C=I$ and $Z D^{+}=0$ have a unique common solution given by $Z=\left(C-D^{+} D C\right)^{+}\left(I-D^{+} D\right)$.

Proof.

$$
\begin{aligned}
Z C & =\left(C-D^{+} D C\right)^{+}\left(I-D^{+} D\right) \\
& =\left(C-D^{+} D C\right)^{+}\left(C-D^{+} D C\right) \\
& =I \\
Z D^{+} & =\left(C-D^{+} D C\right)^{+}\left(I-D^{+} D\right) D^{+} \\
& =\left(C-D^{+} D C\right)^{+}\left(D^{+}-D^{+} D D^{+}\right) \\
& =\left(C-D^{+} D C\right)^{+}\left(D^{+}-D^{+}\right) \\
& =0 .
\end{aligned}
$$

Theorem 4. If $A X=I$ and $B X=0$ then $S(A-X B)$ is hermitian iff $X=A B^{+}$where $P=\left[\begin{array}{c}A \\ B\end{array}\right]$ and $P^{-1}=[S T]$.

Proof. First suppose that $S(A-X B)$ is hermitian, then

$$
\begin{array}{r}
S(A-X B)=[S(A-X B)]^{*}=(A-X B)^{*} S^{*} \\
A(S(A-X B)) B^{*}=A(A-X B)^{*} S^{*} B^{*}=A(A-X B)^{*}(B S)^{*} . \\
\text { Now } P P^{-1}=\left[\begin{array}{l}
A \\
B
\end{array}\right]\left[\begin{array}{ll}
S & T
\end{array}\right]=\left[\begin{array}{cc}
A S & A T \\
B S & B T
\end{array}\right]=\left[\begin{array}{ll}
1 & 0 \\
0 & 1
\end{array}\right] \\
\therefore A S=B T=I \text { and } A T=B S=0, \text { so, }(B S)^{*}=0 .
\end{array}
$$

Thus (2) gives

or,

$$
\begin{aligned}
& A(S(A-X B)) B^{*}=0 \\
& A S(A-X B)) B^{*}=0
\end{aligned}
$$




$$
\begin{array}{ll}
\text { or, } & I(A-X B) B^{*}=0 \\
\text { or, } & (A-X B) B^{*}=0 \\
\text { or, } & A B^{*}-X B B^{*}=0 \\
\text { or, } & X B B^{*}=A B^{*} .
\end{array}
$$

Let $B B^{*}$ be non-singular, then $\left(\left(B B^{*}\right)^{-1}\right.$ exists and

$$
X=A B^{*}\left(B B^{*}\right)^{-1}=A B^{+}=A B^{+} \text {, since } B^{*}\left(B B^{*}\right)^{-1}=B^{+} \text {. }
$$

Conversely, $\quad$ if $X=A B^{+}$then $S(A-X B)$ is hermitian.

Because $S(A-X B)=S\left(A-A B^{+} B\right)$ and $A S=I$ and $B S=0$ have a unique common solution given by

$$
\begin{aligned}
S= & \left(I-B^{+} B\right)\left(A-A B^{+} B\right)^{+} \\
\therefore \quad S(A-X B) & =\left(I-B^{+} B\right)\left(A-A B^{+} B\right)^{+}\left(A-A B^{+} B\right) \\
{[S(A-X B)]^{*} } & =\left[\left(I-B^{+} B\right)\left(A-A B^{+} B\right)^{+}\left(A-A B^{+} B\right)\right]^{*} \\
= & {\left[\left(A-A B^{+} B\right)^{*}\left[\left(A-A B^{+} B\right)^{+}\right]^{*}\left(I-B^{+} B\right)^{*}\right.} \\
& =\left[\left(A-A B^{+} B\right)^{+}\left(A-A B^{+} B\right)\right]^{*}\left(I-B^{+} B\right) \\
\text { since }\left(I-B^{+} B\right) \text { is hermitian. } & \\
& =\left(A-A B^{+} B\right)^{+}\left(A-A B^{+} B\right)\left(I-B^{+} B\right) .
\end{aligned}
$$

Theorem 5. If $Z C=I$ and $Z D=0$ then $(C-D Z) L$ is hermitian iff $Z=D^{+} C$ where $Q=[C D]$ and $Q^{-1}=\left[\begin{array}{c}L \\ M\end{array}\right]$.

Proof. First suppose that $(C-D Z) L$ is hermitian then

$$
\begin{aligned}
& (C-D Z) L=[(C-D Z) L\}]^{*}=L^{*}(C-D Z)^{*} \\
& D^{*}((C-D Z) L) C=D^{*} L^{*}(C-D Z)^{*} C=(L D)^{*}(C-D Z)^{*} C . \\
& \text { Now } \quad Q^{-1} Q=\left[\begin{array}{c}
L \\
M
\end{array}\right][C D]=\left[\begin{array}{cc}
L C & L D \\
M C & M D
\end{array}\right]=\left[\begin{array}{ll}
1 & 0 \\
0 & 1
\end{array}\right] \\
& \therefore L C=M D=I \text { and } L D=M C=0 . \text { So, }(L D)^{*}=0 .
\end{aligned}
$$

Thus (3) gives

or,

or,

or,

or,

or,

Let,

$$
\begin{gathered}
D^{*}((C-D Z) L) C=0 \\
D^{*}(C-D Z) L C=0 \\
D^{*}(C-D Z) I=0 \\
D^{*}(C-D Z)=0 \\
D^{*} C-D^{*} D Z=0 \\
D^{*} D Z=D^{*} C .
\end{gathered}
$$

$D^{*} D$ be non-singular then $\left(D^{*} D\right)^{-1}$ exists and 


$$
Z=\left(D^{*} D\right)^{-1} D^{*} C=D^{+} C \text { since }\left(D^{*} D\right)^{-1} D^{*}=D^{+}
$$

Conversely, $\quad$ if $Z=D^{+} C$ then $(C-D Z) L$ is hermitian.

Because $\quad(C-D Z) L=\left(C-D D^{+} C\right) L$ and

$L C=I$ and $L D=0$ have a unique common solution given by

$$
\begin{gathered}
L=\left(C-D D^{+} C\right)^{+}\left(I-D D^{+}\right) \\
\therefore(C-D Z) L=\left(C-D D^{+} C\right)\left(C-D D^{+} C\right)^{+}\left(I-D D^{+}\right) .
\end{gathered}
$$

Also

$$
\begin{aligned}
{[(C-D Z) L]^{*} } & =\left[\left(C-D D^{+} C\right)\left(C-D D^{+} C\right)^{+}\left(I-D D^{+}\right)\right]^{*} \\
& =\left[\left(I-D D^{+}\right)^{*}\left[\left(C-D D^{+} C\right)^{+}\right]^{*}\left(C-D D^{+} C\right)^{*}\right. \\
& \text { since }\left(I-D D^{+}\right) \text {is hermitian. } \\
& =\left[\left(I-D D^{+}\right)\left(C-D D^{+} C\right)^{+}\left(C-D D^{+} C\right)^{*} .\right.
\end{aligned}
$$

Theorem 6. Consistent equations $A x=y$ have a solution $x=A^{-} y$ iff $A A^{-} A=A$.

Proof. If $A x=y$ are consistent and have $x=A^{-} y$ as a solution, write $a_{i}$ for the i-th column of $A$ and consider the equations $A x=a_{i}$. They have a solution, the null vector with its $\mathrm{i}$-th element set equal to unity. Therefore, the equations $A x=a_{i}$ are consistent.

Furthermore, since consistent equations $A x=y$ have a solution $x=A^{-} y$, it follows that consistent equations $A x=a_{i}$ have a solution $x=A^{-} a_{i}$ and this is true for all values of $i$, i.e, for all columns of $A$. Hence $A A^{-} A=A$.

Conversely, if $A A^{-} A=A$, then $A A^{-} A x=A x$ and when $A x=y$ this gives $A A^{-} y=y$, i.e $A\left(A^{-} y\right)=y$.

Hence $x=A^{-} y$ is a solution of $A x=y$ and the theorem is proved.

Theorem 7. If $A$ has $q$ columns and if $A^{-}$is a generalized inverse of $A$, then the consistent equations $A x=y$ have the solution $\bar{x}=A^{-} y+\left(A^{-} A-I\right) z$, , where $z$ is an arbitrary vector of order $q$.

Proof. We know

$$
\begin{aligned}
A \bar{x} & =A A^{-} y+\left(A A^{-} A-A\right) z \\
& =A A^{-} y, \text { since } A A^{-} A=A \\
& =y ; \text { since } A A^{-} y=y
\end{aligned}
$$

i.e. $\bar{x}$ satisfies $A x=y$ and hence is solution.

Example. We have to find a particular solution and also the general solution of the following system of linear equations by using generalized inverse: 


$$
\begin{aligned}
& 2 x_{1}+3 x_{2}+x_{3}+3 x_{4}=14 \\
& x_{1}+x_{2}+x_{3}+2 x_{4}=6 \\
& 3 x_{1}+5 x_{2}+x_{3}+4 x_{4}=22 .
\end{aligned}
$$

Solution. The given system of linear equations can be written in matrix-form as

(4)

$$
\left(\begin{array}{llll}
2 & 3 & 1 & 3 \\
1 & 1 & 1 & 2 \\
3 & 5 & 1 & 4
\end{array}\right)\left(\begin{array}{l}
x_{1} \\
x_{2} \\
x_{3} \\
x_{4}
\end{array}\right)=\left(\begin{array}{c}
14 \\
6 \\
22
\end{array}\right)
$$

Let

$$
A=\left(\begin{array}{llll}
2 & 3 & 1 & 3 \\
1 & 1 & 1 & 2 \\
3 & 5 & 1 & 4
\end{array}\right), x=\left(\begin{array}{c}
x_{1} \\
x_{2} \\
x_{3} \\
x_{4}
\end{array}\right) \text { and } y=\left(\begin{array}{c}
14 \\
6 \\
22
\end{array}\right)
$$

The system (4) can be written as

$$
A x=y \text {. }
$$

First, we will find out the generalized inverse of $A$ for which we need the rank of $A$. Reduce the matrix $A$ to row -echelon form by the elementary row operations.

$$
\begin{aligned}
A & =\left(\begin{array}{llll}
2 & 3 & 1 & 3 \\
1 & 1 & 1 & 2 \\
3 & 5 & 1 & 4
\end{array}\right) \\
& \sim\left(\begin{array}{cccc}
1 & 1 & 1 & 2 \\
2 & 3 & 1 & 3 \\
3 & 5 & 1 & 4
\end{array}\right) \\
\sim & \left(\begin{array}{cccc}
1 & 1 & 1 & 2 \\
0 & 1 & -1 & -1 \\
0 & 2 & -2 & -2
\end{array}\right) \\
\sim & \sim\left(\begin{array}{cccc}
1 & 1 & 1 & 2 \\
0 & 1 & -1 & -1 \\
0 & 0 & 0 & 0
\end{array}\right)
\end{aligned}
$$

This matrix is in row echelon form and has two non-zero rows. So, rank of $A$ is 2 . Now let us partition the matrix $A$ in the following way: 


$$
\begin{aligned}
A & =\left(\begin{array}{ll|ll}
2 & 3 & 1 & 3 \\
1 & 1 & 1 & 2 \\
\hline 3 & 5 & 1 & 4
\end{array}\right) \\
& =\left(\begin{array}{ll}
A_{11} & A_{12} \\
A_{21} & A_{22}
\end{array}\right) \text { where } A_{11}=\left(\begin{array}{ll}
2 & 3 \\
1 & 1
\end{array}\right)
\end{aligned}
$$

Since, $\left|A_{11}\right|=\left|\begin{array}{ll}2 & 3 \\ 1 & 1\end{array}\right|=2-3=-1 \neq 0$

$$
A_{11}^{-1} \text { exists and } A_{11}^{-1}=\left(\begin{array}{cc}
-1 & 3 \\
1 & -2
\end{array}\right) \text {. }
$$

Hence a g-inverse of $A$ is

$$
A^{-}=\left(\begin{array}{ccc}
-1 & 3 & 0 \\
1 & -2 & 0 \\
0 & 0 & 0 \\
0 & 0 & 0
\end{array}\right)
$$

Thus a particular solution of the system is

$$
\begin{gathered}
x=\left(\begin{array}{l}
x_{1} \\
x_{2} \\
x_{3} \\
x_{4}
\end{array}\right)=\left(\begin{array}{ccc}
-1 & 3 & 0 \\
1 & -2 & 0 \\
0 & 0 & 0 \\
0 & 0 & 0
\end{array}\right)\left(\begin{array}{c}
14 \\
6 \\
22
\end{array}\right)=\left(\begin{array}{l}
4 \\
2 \\
0 \\
0
\end{array}\right) . \\
\therefore x_{1}=4, x_{2}=2, x_{3}=0, x_{4}=0 .
\end{gathered}
$$

Now we will find out the general solution of the given system.

When $A$ has $q$ columns and $A^{-}$is a generalized inverse of $A$, then the consistent system $A x=y$ have solutions $x=A^{-} y+\left(A^{-} A-I\right) z$, where $z$ is any arbitrary vector of order $q$.

$$
A^{-} A-I=\left(\begin{array}{ccc}
-1 & 3 & 0 \\
1 & -2 & 0 \\
0 & 0 & 0 \\
0 & 0 & 0
\end{array}\right)-\left(\begin{array}{cccc}
2 & 3 & 1 & 3 \\
1 & 1 & 1 & 2 \\
3 & 5 & 1 & 4
\end{array}\right)
$$




$$
=\left(\begin{array}{cccc}
0 & 0 & 2 & 3 \\
0 & 0 & -1 & -1 \\
0 & 0 & -1 & 0 \\
0 & 0 & 0 & -1
\end{array}\right)
$$

Therefore, the general solution of the linear system is

$$
\begin{aligned}
& x= A^{-} y+\left(A^{-} A-I\right) z, \\
&=\left(\begin{array}{l}
4 \\
2 \\
0 \\
0
\end{array}\right)+\left(\begin{array}{cccc}
0 & 0 & 2 & 3 \\
0 & 0 & -1 & -1 \\
0 & 0 & -1 & 0 \\
0 & 0 & 0 & -1
\end{array}\right)\left(\begin{array}{l}
z_{1} \\
z_{2} \\
z_{3} \\
z_{4}
\end{array}\right)=\left(\begin{array}{l}
4 \\
2 \\
0 \\
0
\end{array}\right)+\left(\begin{array}{c}
2 z_{3}+3 z_{4} \\
-z_{3}-z_{4} \\
-z_{3} \\
-z_{4}
\end{array}\right) \\
&\left(\begin{array}{l}
x_{1} \\
x_{2} \\
x_{3} \\
x_{4}
\end{array}\right)=\left(\begin{array}{c}
4+2 z_{3}+3 z_{4} \\
2-z_{3}-z_{4} \\
-z_{3} \\
-z_{4}
\end{array}\right) \\
& x_{1}=4+2 z_{3}+3 z_{4} \\
& x_{2}=2-z_{3}-z_{4} \\
& x_{3}=-z_{3} \\
& x_{4}=-z_{4}
\end{aligned}
$$

\section{References:-}

1. M. M. Ahmed, Unified approach of generalized inverse and its applications, BRAC Univ. J. III (2) (2006), 4145.

2. F. A. Graybill, Matrices with applications in statistics, The Wadsworth Statistics/ Probability Series, Wadsworth International Group, Belmont, California, 1969.

3. E. H. Moore, On the reciprocal of the general algebraic matrix, Abst. Bull. Amer. Math. Soc. (1920), 304-305.

4. R. Penrose, A generalized inverse for matrices, Proc. Cambridge Philo. Soc. 51 (1955), 406-413.

5. C. R. Rao and S. K. Mitra, Generalized Inverse of Matrices and its Applications, Wiley, New York, 1971.

6. C. A. Rohde, Some results on generalized inverses, SIAM Rev. 8(2) (1966), 201- 205. 